\title{
REE Patterns in Ca-Al Rich Phases of CV Chrondrite Meteorites
}

\author{
Julia C. GonZales ${ }^{1,2}$, Marina E. Gemma ${ }^{2,3}$, \\ DENTON S. EBEL ${ }^{2}$
}

${ }^{1}$ Dept. of Earth, Planetary, and Space Sciences, University of California, Los Angeles, Los Angeles, CA, 90024.

${ }^{2}$ Dept. of Earth and Planetary Sciences, American Museum of Natural History, New York, NY, 10024.

${ }^{3}$ Dept. of Earth and Environmental Sciences, Columbia

University, New York, NY, 10027.

*correspondence: [juliacaitlin@ucla.edu]

Carbonaceous chondrites are the most primitive type of meteorite, forming soon after the sun in the protoplanetary disk. They are highly pristine, preserving the conditions that existed concurrent to their formation. The distributions of trace elements among the mineral phases and throughout the inclusions in these chondrites help to constrain early solar system conditions [1]. Refractory constituents such as Ca-Alrich inclusions (CAIs) and chondrule mesostasis (glass) are expected to host most of the refractory REEs. However, these elements are also found in mineral phases that form at lower temperatures $[2,3]$. This work evaluates the REE patterns in refractory components for multiple sections of three $\mathrm{CV}$ chondrites (Allende, Leoville, and Vigarano).

First, ten X-ray element intensity maps of each section were made using an electron microprobe. Combinations of three element maps were used to create composite images to reveal qualitative, compositional information about the sample's inclusions, from which mineral phases and target spots for quantitative analysis were identified. REE abundances for each spot were measured using a Laser Ablation-Inductively Coupled Plasma-Mass Spectrometer. A total of 126 spots were analyzed, ranging in size from 20 to 85 microns. The data was processed using three different normalizing masses $(\mathrm{Ca}, \mathrm{Mg}$, and $\mathrm{Si}$ ) which were uniquely chosen for each spot, depending on the mineral phases present. Preliminary results show that REE patterns in chondrule glasses are relatively flat, with values around $10 \mathrm{x}$ chondritic (CI). REE patterns in CV CAIs are more variable, but tend to cluster around $100 \mathrm{x}$ CI values. This work is the first step in a larger effort to characterize REE patterns across all mineral phases in carbonaceous chondrites.

[1] Boyton W. (1984) Developments in Geochemistry, vol. 2, 63-114. [2] Jacquet, E., et. al (2012). Meteor. Planet. Sci. 47, 1695-1714. [3] Alexander, C.M.O'D. (1994) Geochim. Cosmochim. Acta, 58, 3451-3467. 